\title{
The diagnostic value of perivascular infiltrates in muscle biopsy specimens for the assessment of rheumatoid vasculitis
}

\author{
Alexandre E Voskuyl, Sjoerd G van Duinen, Aeilko H Zwinderman, \\ Ferdinand C Breedveld, Johanna M W Hazes
}

\begin{abstract}
Objective-To determine the diagnostic value of perivascular infiltrates (PVI) in randomly obtained muscle biopsy specimens for the assessment of rheumatoid vasculitis (RV).

Methods-The number and size of PVIs, defined as the presence of mononuclear or polymorphonuclear cells around $\geqslant 50 \%$ of the circumference of a vessel wall, as well as the presence of fibrinoid necrosis were determined in frozen sections of muscle samples of RV patients with histologically confirmed vasculitis in fixed muscle tissue $(n=12)$. The findings were compared with those observed in frozen sections of muscle biopsy specimens of rheumatoid arthritis (RA) patients not suspected of vasculitis $(n=14)$ and patients with osteoarthritis (OA) $(n=11)$. The presence of PVIs and of fibrinoid necrosis were sought in four frozen sections of the muscle biopsy specimen.
\end{abstract}

Results-PVIs were observed in $75 \%$ of the RV patients, which was significantly (p < $0.05)$ higher than the frequency found in RA $(14 \%)$ or OA $(18 \%)$ patients. PVIs with $\geqslant$ three cell layers were found in $67 \%$ of the RV patients and in none of the RA and OA patients $(p<0.05)$. Fibrinoid necrosis was found in least one of four frozen section in $33 \%$ of the RV patients. There was a good intra-observer and interobserver concordance on the presence of fibrinoid necrosis and of PVIs with $\geqslant$ three cell layers.

Conclusions-The assessment of PVIs with $\geqslant$ three cell layers in a muscle biopsy specimen is a specific and reliable test in discriminating RV from RA without vasculitis. The demonstration in muscle of PVIs with $\geqslant$ three cell layers is more sensitive than that of fibrinoid necrosis in the diagnosis of RV.

(Ann Rheum Dis 1998;57:114-117)

and Medical Statistics A $\mathrm{H}$ Zwinderman

University Hospital Leiden, Leiden, the Netherlands

Correspondence to: Dr A E Voskuyl, Department of Rheumatology, Room B-417, University Hospital Vrije Universiteit, PO Box 7057, 1007 MB Amsterdam, the Netherlands.

Accepted for publication 17 December 1997 transmural cellular infiltrate within the vessel wall. This definition is in line with the ous clinical presentation. ${ }^{1-3}$ Medium sized arteries, arterioles, venules, and capillaries can be affected. ${ }^{4}{ }^{5}$ Histologically, RV is generally infiltrate with destruction of the vessel wall. ${ }^{4}$ The destruction is defined by the observation histological criteria of other vasculitic syndromes, such as polyarteritis nodosa and hypersensitivity vasculitis. ${ }^{7}$

The diagnosis RV is generally pursued by histological examination of a biopsy specimen of affected organs. ${ }^{38}$ When RV is suspected and affected organs cannot be biopsied, histological examination of muscle or rectum samples has been advocated. ${ }^{3910}$ For the assessment of vasculitis, however, the diagnostic yield of a muscle biopsy specimen is considered to be less than $50 \% .{ }^{11}{ }^{12}$ This low sensitivity is ascribed both to segmental distribution of vasculitis within the muscle and to restricted organ involvement. ${ }^{5}$

In RV patients, perivascular infiltrates (PVI) with mono/polymorphonuclear cells are regularly observed in both normal and abnormal vessels. ${ }^{6}$ The perivascular inflammatory cells are arranged in layers that vary in number and size. Previous observations in skin biopsy specimens report that the number and size of PVI are greater in the non-lesional skin of RV patients when compared with rheumatoid arthritis (RA) patients without vasculitis. ${ }^{13}$ Our experience with muscle biopsy specimens suggested that PVIs occur more frequently than fibrinoid necrosis in RA patients suspected of vasculitis.

This study was designed to evaluate whether the presence and the size of PVIs in muscle biopsy specimens might contribute to the diagnosis of RV. This was done by comparing standard assessment of muscle biopsy specimens of two contrasting patient groups: (1) RA patients with clinical and histological evidence of vasculitis, (2) RA patients and patients with osteoarthritis (OA) without clinical and histological evidence of vasculitis.

\section{Methods}

PATIENTS

Twelve RA patients with clinical symptoms and histological evidence of vasculitis were studied as RV patients. These patients were selected when fibrinoid necrosis was observed during extensive histological examination of the rectus femoris muscle biopsy specimen. The RV patients, five female and seven male, had a median age of 70 years (range 54-81) and a median RA disease duration of 20 years (range 1-52). All RV patients had increased serum concentrations of rheumatoid factors during their RA history, and $67 \%$ had rheumatoid nodules. At the time RV was diagnosed five of the patients were being treated with disease 
Table 1 Clinical signs of patients with rheumatoid vasculitis

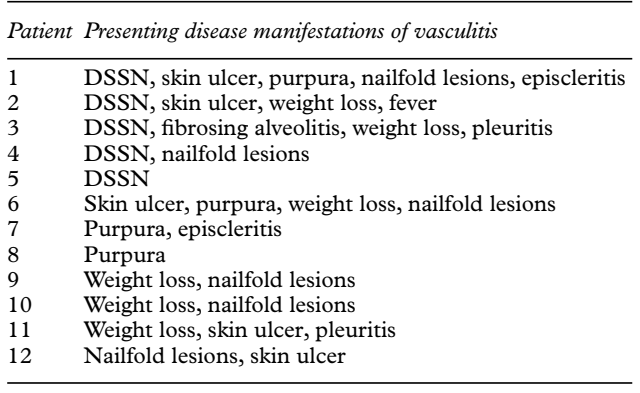

DSSN $=$ distal sensory-motor or sensoric neuropathy.

modifying anti-rheumatic drugs (DMARDs), and two with corticosteroids. Table 1 shows the clinical signs that led to the suspicion of vasculitis in the RV group.

In addition, $14 \mathrm{RA}$ patients and $11 \mathrm{OA}$ patients were studied as controls. A sample of the rectus femoris muscle of these patients was obtained during surgery for a total hip or knee arthroplasty. The RA control patients, 10 female and four male, had a median age of 70 years (range 56-86) and a median RA disease duration of 12 years (range 1-32). Thirteen of 14 RA patients had increased serum concentrations of rheumatoid factors during their RA history, and $43 \%$ had rheumatoid nodules. At the time of the muscle biopsy, nine of the RA patients were treated with DMARDs, but none with corticosteroids. The OA patients, eight female and three male, had a median age of 70 years (range 52-87). None was treated with DMARDs or corticosteroids. None of the patients with RA or OA had histological evidence of vasculitis on extensive histological examination of the muscle biopsy specimen.

All RV and RA patients fulfilled the 1987 American Rheumatism Association criteria for RA. Informed consent was given by all patients. The study protocol was approved by the Committee of Medical Ethics of the University Hospital Leiden.

\section{MUSCLE TISSUE}

The muscle sample of all patients was obtained according to a predefined protocol by an open surgical procedure from a (non-symptomatic) rectus femoris muscle. The muscle sample was divided into two parts. One part was used for extensive histological examination; in the absence of fibrinoid necrosis in the first series of sections, a total of 45 sections were subsequently analysed for the presence of fibrinoid necrosis. The sections were cut and stained with haematoxylin and eosin. The other part of the muscle tissue was snap frozen in Tissue-Tek OCT (Miles Inc-Diagnostic Division, Elkhart, IN) by immersion in methylbutane $\left(-70^{\circ} \mathrm{C}\right)$. Frozen blocks were stored at $-70^{\circ} \mathrm{C}$ until sectioned for staining. Serial sections of $7 \mu \mathrm{m}$ were cut on a cryostat and placed on glass slides (Star Frost adhesive slides, Knittelgläser, Germany). From these frozen sections, four sections were chosen randomly at a regular interval of $80 \mu \mathrm{m}$ and stained with haematoxylin and eosin.
MICROSCOPICAL ANALYSIS

Haematoxylin and eosin stained sections of the frozen blocks were coded and analysed randomly. The area of each section was measured and the histological features were scored by two independent observers, who were unaware of the diagnosis. One observer (AEV) scored the sections twice with an interval of two weeks.

The presence of fibrinoid necrosis, the number and the size of PVIs were assessed in each frozen muscle section. Fibrinoid necrosis was defined as the observation of amorphous eosinophilic material within a vessel wall. A PVI was defined as the presence of mono/ polymorphonuclear cells around, at least $50 \%$, of the circumference of a vessel wall. A vessel should be clearly visible, as defined by the presence of a lumen surrounded by a vessel wall. The size of a PVI was defined by the number of cell layers that surrounded, at least $50 \%$, of the circumference of a vessel wall. Subsequently, each muscle section was classified into a PVI category, according to the highest number of cell layers observed. Three categories of PVIs were discerned: $1=$ at most one cell layer; $2=$ at most two cell layers; $3=3$ or more cell layers.

\section{STATISTICAL ANALYSIS}

Comparison of the muscle samples between the RV, RA, and OA patient were done for: (1) the presence of PVIs and for the PVI category using the $\chi^{2}$ test; (2) the total number of PVIs and the median surface area of the four sections using the Kruskal-Wallis test and the Mann-Whitney U test. The sensitivity and specificity in discriminating RV from non-RV was calculated for the presence of PVIs and for the various PVI categories. The intra and interobserver agreements on the presence of fibrinoid necrosis and PVIs were estimated by Cohen's $\kappa$, and by Spearman's rank correlation for the category of PVI. $p$ Values less than 0.05 were considered significant.

\section{Results}

The median surface area of the four frozen muscle sections of RV patients $\left(43 \mathrm{~mm}^{2}\right.$; range 19-110 $\mathrm{mm}^{2}$ ) was not significantly different from that of RA patients $\left(42 \mathrm{~mm}^{2}\right.$; range $24-77$ $\mathrm{mm}^{2}$ ) or of OA patients $43 \mathrm{~mm}^{2}$; range $21-121$ $\left.\mathrm{mm}^{2}\right)$.

FIBRINOID NECROSIS

In the fixed muscle sections fibrinoid necrosis was observed in all RV patients and in none of the RA or OA patients. When considering just the four frozen sections, fibrinoid necrosis was observed in four of $12 \mathrm{RV}$ patients and in none of the RA or OA patients (table 2). The results presented are those as scored at the first examination of one observer (AEV). Fibrinoid necrosis was always accompanied by a PVI around the same vessel (fig 1(D)). The intra and inter-observer Cohen's $\kappa$ agreements on the presence of fibrinoid necrosis in the four muscle frozen sections were 1.0 (SEM 0.16) and 0.87 (SEM 0.12), respectively. The only 
Table 2 Presence of fibrinoid necrosis and perivascular cellular infiltrates (PVI) in four spaced frozen sections of a muscle biopsy specimen as assessed in patients with rheumatoid vasculitis $(R V)$, rheumatoid arthritis $(R A)$, and primary osteoarthritis $(O A)$

\begin{tabular}{|c|c|c|c|}
\hline & $\begin{array}{l}R V \\
(n=12)\end{array}$ & $\begin{array}{l}R A \\
(n=14)\end{array}$ & $\begin{array}{l}O A \\
(n=11)\end{array}$ \\
\hline Fibrinoid necrosis present & $4(33)^{\star}$ & 0 & 0 \\
\hline PVI present & $9(75)+$ & $2(14)$ & $2(18)$ \\
\hline Median of (range) & $6(0-4) \ddagger$ & $0(0-2)$ & $0(0-2)$ \\
\hline $\begin{array}{l}\text { PVI of one or two cell layers } \\
\text { present }\end{array}$ & $1(8)$ & $2(14)$ & $2(18)$ \\
\hline PVI of $\geqslant 3$ cell layers present & $8(67) \dagger$ & 0 & 0 \\
\hline
\end{tabular}

${ }^{\star}$ Figures represent number of patients, percentages in parentheses (unless otherwise stated). +RV $v$ RA and OA; $p<0.05, \chi^{2}$ test. $\ddagger R V v R A$ and $\mathrm{OA} ; \mathrm{p}<0.05$, Mann-Whitney U test. The results presented are those as scored at the first microscopic analysis of one observer in four frozen sections of one muscle sample. All RV patients had histological evidence of vasculitis in fixed muscle tissue.

inter-observer disagreement concerned the presence of fibrinoid necrosis in one muscle section of a RV patient.

\section{PRESENCE OF PERIVASCULAR INFILTRATES}

PVIs were observed significantly more frequently in the muscle sections of the RV patients when compared with those of the RA or OA patients (table 2). The number of PVIs was also found to be higher in the muscle sections of RV patients when compared with those of both RA and OA patients (table 2). PVIs were found around vessels with and without fibrinoid necrosis. In the muscle sections without fibrinoid necrosis, PVIs were also found more frequently in RV patients when compared with RA and OA patients. PVIs consisted mainly of mononuclear cells (fig 1). Polymorphonuclear cells were observed in small numbers and only in the muscle sections of RV patients with fibrinoid necrosis (data not shown). The intra and inter-observer Cohen's $\kappa$ agreements on the presence of PVIs were 0.82 (SEM 0.09) and 0.63 (SEM 0.14), respectively. The sensitivity and specificity of the presence of PVIs in discriminating RV from non-RV were $75 \%$ and $86 \%$, respectively.
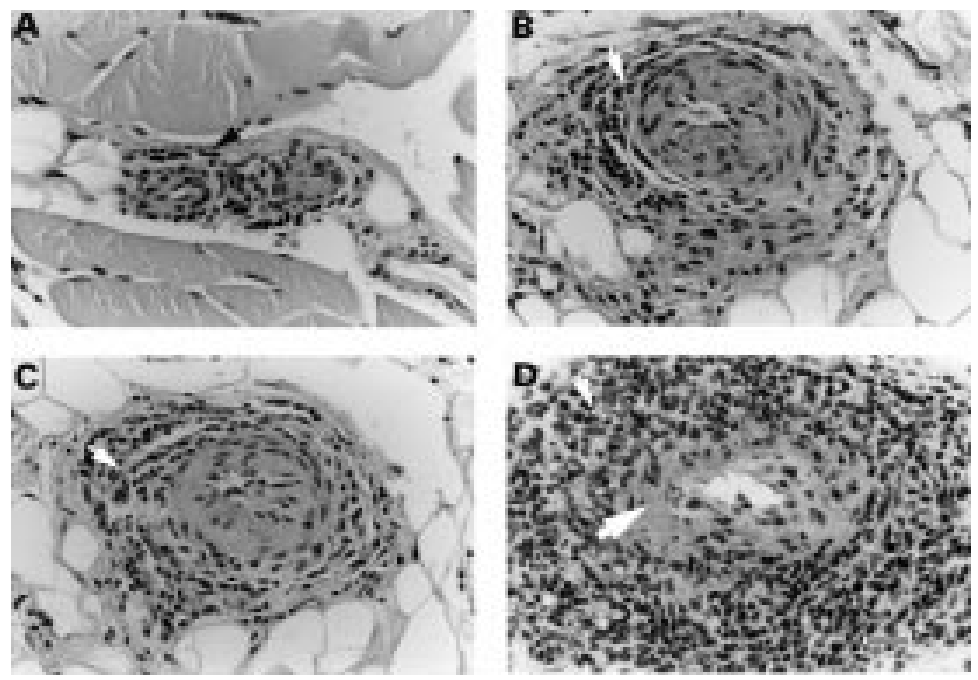

Figure 1 (A) PVI with one cell layer (arrow): at most one cell layer of mononuclear cells surrounding at least $50 \%$ of the vessel wall. (B) PVI with two cell layers (arrow): at most two cell layers of mononuclear cells surrounding at least $50 \%$ of the vessel wall. (C) PVI with $\geq$ three cell layers (arrow): three cell layers of mononuclear cells surrounding at least $50 \%$ of the vessel wall. (D) Fibrinoid necrosis (large arrow), PVI with $\geq$ three cell layers (small arrow). Haematoxylin and eosin stain; original magnification $\times 400$, bar $=40 \mu \mathrm{m}$.
CATEGORY OF PERIVASCULAR INFILTRATE

PVIs with one or two cell layers were observed in the muscle sections of all patient groups (table 2). PVIs with $\geqslant$ three cell layers were only observed in the muscle sections of $\mathrm{RV}$ patients (table 2). In the 32 frozen muscle sections of the eight RV patients in whom PVIs with $\geqslant$ three cell layers were observed, $11 \mathrm{sec}-$ tions showed no PVIs with $\geqslant$ three cell layers nor fibrinoid necrosis, 11 sections showed PVIs with $\geqslant$ three cell layers as well as fibrinoid necrosis, and 10 sections showed PVIs with $\geqslant$ three cell layers but no fibrinoid necrosis. In the 16 frozen muscle sections of the four RV patients in whom PVIs with $\geqslant$ three cell layers was absent, two sections showed PVIs of one or two cell layers and 14 sections showed no PVIs. In addition, none of those 16 sections showed fibrinoid necrosis.

The intra and inter-observer agreements on the presence of PVIs $\geqslant$ three cell layers as assessed by the Spearman rank correlation were 0.92 (SEM 0.93) and 0.84 (SEM 0.10), respectively. The intra and inter-observer disagreement on the number of cell layers consisted of a maximum variation of one cell layer. The intra and inter-observer disagreement on the presence of PVIs $\geqslant$ three cell layers concerned one and two RV patients, respectively. Disagreement did not occur in the RA or OA groups. Figure 1 shows examples of PVI categories. The sensitivity and specificity of PVIs $\geqslant$ three cell layers in discriminating RV from non-RV was $67 \%$ and $100 \%$, respectively.

\section{Discussion}

The results of this study show that the presence of a PVI with $\geqslant$ three cell layers in a muscle biopsy specimen is a highly specific and reliable test, and when compared with the presence of fibrinoid necrosis, a more sensitive marker for the diagnosis RV.

Fibrinoid necrosis was chosen as the histological hallmark of RV. Transmural infiltration of the vessel wall is another morphological abnormality associated with vasculitis. As previously noted, ${ }^{6}$ transmural infiltrates are difficult to determine in muscle biopsy specimens because of the small calibre of the vessels. Hence, transmural infiltrates were not investigated separately in this study.

PVIs have been reported in early studies on muscle biopsy specimens of RV patients. ${ }^{6}$ Such infiltrates have also been found in $26-61 \%$ of the muscle samples of patients with uncomplicated RA, and included, in addition to PVIs around arteries and veins, diffuse interstitial, focal or nodular cellular infiltrates. ${ }^{46-19}$ The results of these studies suggest that cellular infiltrates in muscle biopsy specimens of RA patients are not specific for vasculitis. However, the results of this study suggest that multiple large PVIs in muscle biopsy specimens are associated with the presence of RV. This association could not be explained by the presence of any of the concurrent diseases previously reported to be associated with cellular infiltrates in muscle biopsy specimens, namely myositis, infectious diseases, malignant hypertension or arteriolosclerosis. ${ }^{4}{ }^{14-19}$ 
It should be noted that the presence of PVIs with $\geqslant$ three cell layers was assessed in patients with vasculitis histologically confirmed (fibrinoid necrosis) in the same muscle biopsy specimen. At present, the sensitivity of PVIs with $\geqslant$ three cell layers for RV is unknown in patients without histological evidence of fibrinoid necrosis in a muscle biopsy specimen. Following this study, however, we reviewed the histological features in the muscle biopsy specimens of eight RV patients without vasculitis histologically confirmed in a muscle biopsy specimen. In those patients vasculitis was observed in a biopsy specimen of the skin or sural nerve. Re-examination of the muscle biopsy specimens showed that six of eight specimens had PVIs with $\geqslant$ three cell layers, which is consistent with the results of this study.

The PVIs in the muscle biopsy specimens of this study consisted predominantly of mononuclear cells. Some authors have referred to this type of morphological change as lymphocytic vasculitis, ${ }^{20}$ although others have wondered whether lymfocytic vasculitis is a clinicopathological entity as it may occur in a large number of dermatological diseases. ${ }^{21}$ Anyhow, the results of this study suggest that PVIs in muscle biopsy specimens are a marker of vasculitis in patients with RA.

The diagnostic yield of a muscle biopsy specimen for the assessment of vasculitis in RA has been hitherto considered to be low, and this has been ascribed to the segmental distribution of vasculitis within the muscle. ${ }^{4}$ In line with the findings of others, ${ }^{6}$ we found that the examination of multiple muscle sections improves the diagnostic yield. For routine examination the number of sections examined is generally lower, and close to the four sections assessed in this study. Despite the limited number of sections assessed, our results show that PVIs with $\geqslant$ three cell layers are found more frequently than fibrinoid necrosis, and suggest that the assessment of PVIs increases the diagnostic value of a muscle biopsy specimen.

Should the presence of a PVI with $\geqslant$ three cell layers become accepted as a diagnostic criterion for RV, then the results of this study imply that a careful review of a limited number of spaced muscle sections would be sufficient to establish the diagnosis. In addition, the absence of large PVIs in a muscle biopsy specimen of RA patients strongly suggests the absence of RV. Furthermore, the assessment of PVIs may also be valuable in patients suspected of other vasculitis syndromes such as periarteritis nodosa.

The study was supported by a grant from 'het Nationaal Reumafonds'.

1 Scott DGI, Bacon PA, Tribe CR. Systemic rheumatoid vasculitis: a clinical and laboratory study of 50 cases. Medicine (Baltimore) 1981;60:288-97.

2 Vollertsen RS, Conn DL, Ballard DJ, Ilstrup DM, Kazmar RE, Silverfield JC. Rheumatoid vasculitis: survival and associated risk factors. Medicine (Baltimore) 1986;65: 365-75.

3 Voskuyl AE, Zwinderman AH, Westedt ML, Vandenbroucke JP, Breedveld FC, Hazes JMW. Factors associated with the development of vasculitis in rheumatoid arthritis; with the development of vasculitis in rheumatoid arthritis; results

4 Sokoloff L, Wilens SL, Bunim JJ. Arteritis of striated muscle in rheumatoid arthritis. Am J Pathol 1951;27:157-73.

5 Lie JT. Diagnostic histopathology of major systemic and pulmonary vasculitic syndromes. Rheum Dis Clin N Am 1990;16:269-92.

6 Sokoloff L, Bunim JJ. Vascular lesions in rheumatoid arthritis. J Chron Dis 1957;5: 668-87.

7 Jennette JC, Falk RJ, Andrassy K, Bacon PA, Churg J, Gross WL, et al. Nomenclature of systemic vasculitides: proposal of an international consensus conference. Arthritis Rheum 1994;37:187-92

8 Westedt ML, Daha MR, de Vries E, Valentijn RM, Cats A. IgA containing immune complexes in rheumatoid vasculitis and in active rheumatoid disease. J Rheumatol 1985;12: 449-55.

9 Tribe CR, Scott DGI, Bacon PA. Rectal biopsy in the diagnosis of systemic vasculitis. J Clin Pathol 1981;34:843-50.

10 Voskuyl AE, Zwinderman AH, Westedt ML, Vandenbroucke JP, Breedveld FC, Hazes JMW. The mortality of rheumatoid vasculitis compared with rheumatoid arthritis. rheumatoid vasculitis compared with

11 Maxeiner SR, McDonald JR, Kirklin JW. Muscle biopsy in the diagnosis of periarteritis nodosa. Surgical Clinics of North America 1952;32:1225-33.

12 Dahlberg PJ, Lockhart JM, Overholt EL. Diagnostic studies for systemic necrotizing vasculitis. Arch Intern Med 1989; 149:161-5.

3 Westedt ML, Meijer CJLM, Vermeer BJ, Cats A, de Vries E. Rheumatoid arthritis. The clinical significance of histo-and immunopathological abnormalities in normal skin. J Rheumatol 1984;11:448-53.

14 Sokoloff L, Wilens SL, Bunim JJ. Diagnostic value of histologic lesions of striated muscle in rheumatoid arthritis. Am J Med Sci 1950;219:174-82.

15 Wallace SL, Lattes R, Ragan C Diagnostic significance of the muscle biopsy. Am J Med 1958;25:600-10.

16 Lenoch F, Pazderka V, Hájková Z, Kadlcová L, Poláková. The diagnostic value of muscle biopsy in rheumatoid
The The diagnostic value of muscle biopsy in
arthritis. Acta Rheum Scand 1962;8:103-18.

17 Yates DAH. Muscular changes in rheumatoid arthritis. Ann Rheum Dis 1963;22:342-7.

18 Magyar E, Talerman A, Mohácsy J, Wouters HW, de Bruijn WC. Muscle changes in rheumatoid arthritis. Virchows Arch 1977;373:267-78.

19 Brooke MH, Kaplan H. Muscle pathology in rheumatoid arthritis, polymyalgia rheumatica, and polymyositis. Arch Pathol 1972;94:101-18.

20 Carlson JA, Mihm MC, LeBoit PE. Cutaneous lymphocytic vasculitis: a definition, a review, a proposed classification. Semin Diagn Pathol 1996;13:72-90.

21 Massa MC, Su WPD: Lymphocytic vasculitis: is it a specific entity? J Cutan Pathol 1984;11:132-9. 